Vietnam Journal of Mechanics, VAST, Vol. 29, No. 3 (2007), pp. $221-243$

Special Issue Dedicated to the Memory of Prof. Nguyen Van Dao

\title{
CONSTRUCTION OF ASYMPTOTIC IN KRYLOV-BOGOLIUBOV-MITROPOLSKY SENSE SOLUTION OF WAVE EQUATIONS
}

\author{
Yu. A. Mitropolsky \\ Institute of Mathematics, \\ National Academy of Sciences of Ukraine \\ This article is dedicated to my colleague and intimate friend, \\ who died prematurely being in the prime of life and scientific activity

\begin{abstract}
The paper in question gives consideration to two examples of KBM method for constructing approximate solutions of Klein-Gordon-Bretherton equations often occurred in practice.
\end{abstract}

\section{INTRODUCTION}

Professor, Academician Nguyen Van Dao was tragically killed in Hanoi on December 11, 2006. Nguyen Van Dao was born in August 10, 1937 in the Commune Chi-Tien, District Thanh-Ba, Province Phu-Tho, Vietnam and dedicated all his life to development of science, education and culture of Vietnamese people. Throughout his life Professor Nguyen Van Dao attained great achievements in the field of science and education of Vietnam. He was the President of the Scientific and Education Council of the Vietnam National University of Hanoi; Vice President and General Secretary of the National Center for Scientific Research of Vietnam; Chairman of the Natural Science Council, Ministry of Science and Technology.

Of great importance is his contribution to creation of scientific community to do research, education and application of mechanics in Vietnam. He was the Founding Director of the Institute of Mechanics; Head of the Department, Hanoi University of Technology; President-Founder of the Vietnam Association of Mechanics, the Founding Editor in Chief of the Editorial Board of Vietnam Journal of Mechanics, the Representative of the Vietnam Association of Mechanics in the International Union for Theoretical and Applied Mechanics.

The scientific activity of Professor Nguyen Van Dao was marked by obtaining important results in problems on interaction between nonlinear oscillating systems, non-linear dynamics and chaos as well as asymptotic methods for studying non-linear high degree systems. He published more than 100 scientific works and monographs.

He was also famed for his public activity. He was the member of Presidium of the Central Committee of Vietnam Fatherland Front; Vice-President of Vietnam Peace Committee; President of Association for Liaison with Overseas Vietnamese.

The international activity of Professor Nguyen Van Dao was widely known and highly appreciated in Vietnam and worldwide. He was the Laureate of the Ho Chi Minh Prize 
in the field of Science and Technology, Ukraine National Prize in Science and Technology, the Holder of the 1-st degree Labor Medal, 2-nd degree Resistance Medal. He was elected as the Member of the III-d World Academy of Science; Member of the Czech Academy of Science; Member of Ukraine National Academy of Sciences.

Professor Nguyen Van Dao was exceptionally sweet and hospitable person. I got acquainted with him in 1961 when he first arrived in Kiev to attend the International conference and since then often met with him in Moscow and some cities of Europe including Warsaw, Prague, Berlin and others.

We had close cooperation in the field of mechanics (non-linear mechanics, dynamic systems). Since 1980 I have visited Vietnam 8 times and each arrival was like a festival. Nguyen Van Dao and his wife Chan-Kim-Tyi always met me at the airport, we drove to a hotel where I would stay or they put me up.

The tragic death of Nguyen Van Dao will be immeasurable loss for Vietnamese science. As a scientist and a person he will always be within living memory and my memory in particular. It was Nguyen Van Dao that introduced me to many Vietnamese cities, their lovely sights and hard-working people which I loved so much.

The result of our close cooperation that lasted for more than 30 years was four generalized monographs devoted to nonlinear oscillating systems, numerous participation in various conferences and congresses where we would deliver talks.

\section{FIRST OF ALL WE DEAL WITH THE PROBLEM ON CONSTRUCTING THE ASYMPTOTIC SOLUTION OF PERTURBED KLEIN-GORDON EQUATION}

We consider the equation

$$
\frac{\partial^{2} u}{\partial t^{2}}-C^{2} \frac{\partial^{2} u}{\partial x^{2}}+\lambda^{2} u=\varepsilon f\left(\nu t, u, \frac{\partial u}{\partial t}, \frac{\partial u}{\partial x}\right)
$$

for $\varepsilon=0$, this is the Klein-Gordon equation and, for $\lambda=0$, it turns into the classical wave equation.

Equation (2.1) was studied by many scientists in the course of investigation of nonlinear wave processes in different branches of natural sciences.

Below, we dwell on principal aspects of the application of asymptotic methods of nonlinear mechanics to the construction of approximate solutions of Eq. (2.1). This may be useful for studying special problems of natural sciences that require the investigation of wave processes subject to the action of nonlinear perturbation forces and described by equations of type (2.1). This can also be useful for the analysis of the obtained results. The development and detailed application of the asymptotic method to the solution of a special problem that leads to Eq. (2.1), provided that there is no periodic perturbation with period $\nu$, was first realized in [4].

Thus, for $\varepsilon=0$, Eq. (2.1) is the classical Klein-Gordon wave equation

$$
-C^{2} \frac{\partial^{2} u}{\partial x^{2}}+\lambda^{2} u=0
$$

admitting a solution of the form

$$
u=a \cos \left(k x-\omega_{0} t+\varphi\right),
$$


where $a$ and $\varphi$ are constants and $k$ and $\omega_{0}$ satisfy the variance relation

$$
=C^{2} k^{2}+\lambda^{2} \text {. }
$$

Assume that $\varepsilon>0$ is a small parameter and a function $f\left(\nu t, u, u_{t}, u_{x}\right)$ is periodic (or almost periodic) in $\theta=\nu t$ and has sufficiently many derivatives with respect to the other variables for all their finite values. Denote $u_{t}=\frac{\partial u}{\partial t}, u_{t t}=\frac{\partial^{2} u}{\partial t^{2}}, u_{x}=\frac{\partial u}{\partial x}$, and $u_{x x}=\frac{\partial^{2} u}{\partial x^{2}}$.

Then, according to the well-known statements of the asymptotic method of nonlinear mechanics, we seek an asymptotic solution of Eq. (2.1) for $\varepsilon \rightarrow 0$ in the form of the following series:

$$
\begin{gathered}
u(t, x)=a \cos \psi+\varepsilon u_{1}(a, \psi, \theta)+\varepsilon^{2} u_{2}(a, \psi, \theta)+\ldots \\
\left(\psi=k x-\omega_{0} t+\varphi\right),
\end{gathered}
$$

where $a$ and $\psi$ are determined by the equations

$$
\begin{aligned}
& \frac{\partial a}{\partial t}=\varepsilon A_{1}(a)+\varepsilon^{2} A_{2}(a)+\ldots, \\
& \frac{\partial a}{\partial x}=\varepsilon B_{1}(a)+\varepsilon^{2} B_{2}(a)+\ldots, \\
& \frac{\partial \psi}{\partial t}=-\omega_{0}+\varepsilon C_{1}(a)+\varepsilon^{2} C_{2}(a)+\ldots, \\
& \frac{\partial \psi}{\partial x}=k+\varepsilon D_{1}(a)+\varepsilon^{2} D_{2}(a)+\ldots
\end{aligned}
$$

To simplify calculations, we consider the nonresonance case $(\nu \rightarrow \omega)$.

By differentiating the right-hand sides of series (2.5) and taking (2.6) into account, we obtain expressions for $u_{t}, u_{x}, u_{t t}$, and $u_{x x}$. By substituting these expressions in the left-hand side of Eq. (2.1) and expanding it in powers of $\varepsilon$, we obtain

$$
u_{t t}-C^{2} u_{x x}+\lambda^{2} u=\varepsilon\left\{2\left[\omega_{0} A_{1}+C^{2} k B_{1}\right] \sin \psi\right\}+\varepsilon^{3} .
$$

Further, by substituting the values

$$
\begin{aligned}
u & =a \cos \psi+\varepsilon u_{1}(a, \psi, \theta)+\varepsilon^{2} u_{2}(a, \psi, \theta)+\ldots, \\
u_{t} & =a \omega_{0} \sin \psi+\varepsilon\left\{A_{1} \cos \psi-a \sin \psi C_{1}-u_{1 \psi}^{\prime} \omega_{0}-u_{1 \theta}^{\prime} \nu\right\}+\varepsilon^{2}+\ldots \\
u_{x} & =-a k \sin \psi+\varepsilon\left\{-a \sin \psi D_{1}+u_{1 \psi}^{\prime} k\right\}+\varepsilon^{2} \ldots
\end{aligned}
$$

in the right-hand side of Eq. (2.1) and expanding it in powers of $\varepsilon$, we get

$$
\begin{aligned}
\varepsilon f\left(\theta, u, u_{t}, u_{x}\right)= & \varepsilon f(\theta, a \cos \psi, a \omega \sin \psi,-a k \sin \psi) \\
+ & \varepsilon^{2}\left\{f_{u}^{\prime}\left(\theta, a \cos \psi, a \omega_{0} \sin \psi,-a k \sin \psi\right) u(a, \psi, \theta)\right. \\
+ & f_{u_{t}}^{\prime}\left(\theta, a \cos \psi, a \omega_{0} \sin \psi,-a k \sin \psi\right) \times \\
& \times\left(A_{1} \cos \psi-a C_{1} \sin \psi-u_{1 a}^{\prime} \omega_{0}+u_{1 \theta}^{\prime} \nu\right) \\
& \left.-f_{u_{x}}^{\prime}(\theta, a \cos \psi, a \omega \sin \psi,-a k \sin \psi)\left(a D_{1} \sin \psi-u_{1 \psi}^{\prime} K\right)\right\}+\varepsilon^{3} \ldots
\end{aligned}
$$

By equating the coefficients of the same powers of $\varepsilon$ on the right-hand sides of (2.7) and (2.9) and taking the variance relation (2.4) into account, we obtain the following 
equations for determining $u_{1}(a, \psi, \theta), u_{2}(a, \psi, \theta), \ldots$ :

$$
\begin{aligned}
& \lambda^{2}\left(\frac{\partial^{2} u_{1}}{\partial \psi^{2}}+u_{1}\right)-2 \omega_{0} \nu \frac{\partial^{2} u_{1}}{\partial \psi \partial \theta}+\nu^{2} \frac{\partial^{2} u_{1}}{\partial \theta^{2}} \\
& =-2\left[A_{1} \omega_{0}+C^{2} k B_{1}\right] \sin \psi-2\left[C_{1} \omega_{0}+C^{2} k D_{1}\right] a \cos \psi+f_{0}(\theta, a, \psi), \\
& \lambda^{2}\left(\frac{\partial^{2} u_{2}}{\partial \psi^{2}}+u_{2}\right)-2 \omega_{0} \nu \frac{\partial^{2} u_{2}}{\partial \psi \partial \theta}+\nu^{2} \frac{\partial^{2} u_{2}}{\partial \theta^{2}} \\
& =-2\left[A_{2} \omega_{0}+C^{2} k B_{2}\right] \sin \psi-2\left[C_{2} \omega_{0}+C^{2} k D_{2}\right] a \cos \psi+f_{1}(\theta, a, \psi),
\end{aligned}
$$

where

$$
\begin{aligned}
& f_{0}(\theta, a, \psi)=f\left(\theta, a \cos \psi, a \omega_{0} \sin \psi,-a k \sin \psi\right), \\
& f_{1}(\theta, a, \psi)=f_{u}^{\prime}(\theta, a, \psi) .
\end{aligned}
$$

It is obvious that the functions $f_{0}(\theta, a, \psi), f_{1}(\theta, a, \psi), \ldots$ are $2 \pi$-periodic both in $\psi$ and $\nu t$. In addition, they depend on $a$. The explicit form of these functions is known if the values $A_{j}(a), B_{j}(a), C_{j}(a), D_{j}(a)$, and $u_{j}(a, \psi, \theta), j=1,2, \ldots$, are determined.

Let us determine these functions. For this purpose, we expand the function $f_{0}(a, \psi, \theta)$ in the double Fourier series

$$
f_{0}(a, \psi, \theta)=\sum_{n, m} f_{n, m}^{(0)}(a) e^{i(n \theta+m \psi)}
$$

where

$$
f_{n, m}^{(0)}(a)=\frac{1}{4 \pi^{2}} \int_{0}^{2 \pi} \int_{0}^{2 \pi} f_{0}(a, \psi, \theta) e^{-i(n \theta+m \psi)} d \theta d \psi .
$$

As usual, we seek the function $u_{1}(\theta, a, \psi)$ in the form of the series

$$
u_{1}(\theta, a, \psi)=\sum_{n, m} \bar{f}_{n, m}(a) e^{i(n \theta+m \psi)}
$$

whose coefficients should be defined.

Let us substitute series (2.12) and series (2.14) in Eq. (2.10) and equate the coefficients of the same harmonics on the left-hand and right-hand sides of the obtained expression. Taking into account the condition of absence of zero de nominators, after simple calculation, we obtain the following expression for $u_{1}(\theta, a, \psi)$ :

$$
u_{1}(\theta, a, \psi)=\sum_{\substack{n, m \\(n \neq 0, m \neq 1)}} \frac{f_{n, m}^{(0)}(a) e^{i(n \theta+m \psi)}}{\lambda^{2}\left(1-m^{2}\right)+2 \nu \omega_{0} n m-\nu^{2} n^{2}} .
$$


We also obtain the following expressions for the functions on the right-hand sides of Eqs. (2.6):

$$
\begin{aligned}
& 2\left[A_{1} \omega_{0}+C^{2} k B_{1}\right]=\frac{1}{2 \pi^{2}} \int_{0}^{2 \pi} \int_{0}^{2 \pi} f_{0}(\theta, a, \psi) \sin \psi d \theta d \psi, \\
& 2\left[C_{1} \omega_{0}+C^{2} k D_{1}\right]=\frac{1}{2 \pi^{2} a} \int_{0}^{2 \pi} \int_{0}^{2 \pi} f_{0}(\theta, a, \psi) \cos \psi d \theta d \psi .
\end{aligned}
$$

Hence, taking into account that $y=k x-\omega_{0} t+\varphi$, we obtain, to within values of the first order of smallness,

$$
\begin{aligned}
& \frac{\partial a}{\partial t}+\omega_{0}^{\prime} \frac{\partial a}{\partial x}=\frac{\varepsilon}{4 \pi^{2} \omega_{0}} \int_{0}^{2 \pi} \int_{0}^{2 \pi} f_{0}(\theta, a, \psi) \sin \psi d \theta d \psi, \\
& \frac{\partial \psi}{\partial t}+\omega_{0}^{\prime} \frac{\partial \psi}{\partial x}=\frac{\varepsilon}{4 \pi^{2} \omega_{0} a} \int_{0}^{2 \pi} \int_{0}^{2 \pi} f_{0}(\theta, a, \psi) \cos \psi d \theta d \psi,
\end{aligned}
$$

where $\omega_{0}^{\prime}=d \omega_{0} / d k$ is the so-called group velocity.

By passing to trigonometric functions on the right-hand side of (2.15), we obtain

$$
\begin{aligned}
u_{1}(\theta, a, \psi)= & \frac{1}{2 \pi^{2}} \sum_{\substack{n, m \\
(n \neq 0, m \neq \pm 1)}}\left\{\frac{\cos (n \theta+m \psi)}{\lambda^{2}\left(1-m^{2}\right)+2 \nu \omega_{0} n m-\nu^{2} n^{2}} \times\right. \\
& \times \int_{0}^{2 \pi} \int_{0}^{2 \pi} f_{0}(\theta, a, \psi) \cos (n \theta+m \psi) d \theta d \psi \\
& \left.+\frac{\sin (n \theta+m \psi)}{\lambda^{2}\left(1-m^{2}\right)+2 \nu \omega_{0} n m-\nu^{2} n^{2}} \int_{0}^{2 \pi} \int_{0}^{2 \pi} f_{0}(\theta, a, \psi) \sin (n \theta+m \psi) d \theta d \psi\right\},
\end{aligned}
$$

where $\psi=k x-\omega_{0} t+\varphi$.

We set $u_{x}=u_{x x}=0, \lambda^{2}=\omega^{2}$, and $\omega_{0}=-\omega$ in Eq. (2.1). Then expressions (2.15)(2.18) coincide with the relations presented in [2] for a nonlinear oscillator subject to the action of the perturbation $\varepsilon f\left(\nu t, u, u_{t}\right)$ in the non resonance case.

If the right-hand side of Eq. (2.1) does not depend on $\nu t$, then, instead of (2.17) and (2.18), we get the expressions

$$
\begin{aligned}
& \frac{\partial a}{\partial t}+\omega_{0}^{\prime} \frac{\partial a}{\partial x}=\frac{\varepsilon}{2 \pi \omega_{0}} \int_{0}^{2 \pi} f_{0}(a, \psi) \sin \psi d \psi \\
& \frac{\partial \varphi}{\partial t}+\omega_{0}^{\prime} \frac{\partial \varphi}{\partial x}=\frac{\varepsilon}{2 \pi \omega_{0} a} \int_{0}^{2 \pi} f_{0}(a, \psi) \cos \psi d \psi
\end{aligned}
$$




$$
\begin{gathered}
u_{1}(a, \psi)=\frac{1}{\pi} \sum_{m(m \neq \pm 1)} \frac{1}{\lambda^{2}\left(1-m^{2}\right)}\left\{\cos m \psi \int_{0}^{2 \pi} f_{0}(a, \psi) m \cos \psi d \psi\right. \\
\left.+\sin m \psi \int_{0}^{2 \pi} f_{0}(a, \psi) m \sin \psi d \psi\right\}
\end{gathered}
$$

which coincide with the relations given in [10].

Let us consider in detail the construction of an asymptotic solution of the KleinGordon equation by the Bogolyubov averaging method.

Consider the equation

$$
u_{t t}-C^{2} k u_{x x}+\lambda^{2} u=\varepsilon f\left(u, u_{t}, u_{x}\right)
$$

and assume that its right-hand side satisfies the same conditions as before. For $\varepsilon=0$, Eq. (2.21) has a solution

$$
u=a \cos \psi,
$$

where $\psi=k x-\omega_{0} t+\varphi, a$ and $\varphi$ are arbitrary constants, $\omega_{0}$ and $k$ satisfy the variance relation

$$
\omega_{0}^{2}=C^{2} k^{2}+\lambda^{2}
$$

and the following relations hold:

$$
u_{t}=a \omega_{0} \sin \psi, \quad u_{x}=-a k \sin \psi .
$$

Let us introduce new variables $a(t, x)$ and $\varphi(t, x)$ by the relations

$$
\begin{aligned}
u & =a(t, x) \cos \psi(t, x), \\
u_{t} & =a(t, x) \omega_{0} \sin \psi(t, x),
\end{aligned}
$$

where $\psi(t, x)=k x-\omega_{0} t+\varphi(t, x)$. Then, demanding the expressions (2.25) to be compatible and performing elementary calculations, we get

$$
\begin{aligned}
a_{t} \cos \psi-a \varphi_{t} \sin \psi & =0, \\
a_{x} \cos \psi-a \varphi_{x} \sin \psi & =0 .
\end{aligned}
$$

Multiplying the second relation in (2.26) by $\omega_{0}^{\prime}=C^{2} k / \omega_{0}$ and adding it to the first one, we obtain

$$
\left(a_{t}+\omega_{0}^{\prime} a_{x}\right) \cos \psi-\left(\varphi_{t}+\omega_{0}^{\prime} \varphi_{x}\right) a \sin \psi=0
$$

Further, we have

$$
\begin{gathered}
u_{t t}=a_{t} \omega_{0} \sin \psi-a \omega_{0}^{2} \cos \psi+a \omega_{0} \varphi_{t} \cos \psi, \\
u_{x x}=-a_{x} k_{0} \sin \psi-a k_{0}^{2} \cos \psi-a k_{0} \varphi_{x} \cos \psi
\end{gathered}
$$

Inserting these values in Eq. (2.21), we arrive at the relation

$$
\left(a_{t}+\omega_{0}^{\prime} a_{x}\right) \omega_{0} \cos \psi+\left(\varphi_{t}+\omega_{0}^{\prime} \varphi_{x}\right) \omega_{0} a \sin \psi=\varepsilon f\left(a \cos \psi,+a \omega_{0} \sin \psi,-a k \sin \psi\right) .
$$


It follows from the system of equations (2.27), (2.29) that

$$
\begin{aligned}
& \frac{\partial a}{\partial t}+\omega_{0}^{\prime} \frac{\partial a}{\partial x}=\frac{\varepsilon}{\omega_{0}} f\left(a \cos \psi, a \omega_{0} \sin \psi,-a k \sin \psi\right) \sin \psi, \\
& \frac{\partial \varphi}{\partial t}+\omega_{0}^{\prime} \frac{\partial \varphi}{\partial x}=\frac{\varepsilon}{a \omega_{0}} f\left(a \cos \psi, a \omega_{0} \sin \psi,-a k \sin \psi\right) \cos \psi,
\end{aligned}
$$

where $\psi=k x-\omega_{0} t+\varphi$.

Taking into account that $\omega_{0}^{\prime}=C^{2} k / \omega_{0}$, we write

$$
\begin{gathered}
\omega_{0} \frac{\partial a}{\partial t}=\omega_{0} \frac{\partial a}{\partial \psi} \frac{\partial \psi}{\partial t}=-\omega_{0}^{2} \frac{\partial a}{\partial \psi} \\
C^{2} k \frac{\partial a}{\partial x}=C^{2} k \frac{\partial a}{\partial \psi} \frac{\partial \psi}{\partial x}=C^{2} k^{2} \frac{\partial a}{\partial \psi} .
\end{gathered}
$$

Hence,

$$
\omega_{0}\left(\frac{\partial a}{\partial t}+\omega_{0}^{\prime} \frac{\partial a}{\partial x}\right)=\left(C^{2} k^{2}-\omega_{0}^{2}\right) \frac{\partial a}{\partial \psi}=-\lambda^{2} \frac{\partial a}{\partial \psi} .
$$

Similarly,

$$
\begin{aligned}
\omega_{0} \frac{\partial \varphi}{\partial t} & =\omega_{0} \frac{\partial \varphi}{\partial \psi} \frac{\partial \psi}{\partial t}=\omega_{0}^{2} \frac{\partial \varphi}{\partial \psi} \\
C^{2} k \frac{\partial \varphi}{\partial x} & =C^{2} k \frac{\partial \varphi}{\partial \psi} \frac{\partial \psi}{\partial x}=C^{2} k^{2} \frac{\partial \varphi}{\partial \psi} .
\end{aligned}
$$

Consequently,

$$
\omega_{0}\left(\frac{\partial \varphi}{\partial t}+\omega_{0}^{\prime} \frac{\partial \varphi}{\partial x}\right)=\left(C^{2} k^{2}-\omega_{0}^{2}\right) \frac{\partial \varphi}{\partial \psi}=-\lambda^{2} \frac{\partial \varphi}{\partial \psi} .
$$

Thus, the system of equations (2.30) can be written in the form

$$
\begin{aligned}
& \frac{\partial a}{\partial \psi}=-\frac{\varepsilon}{\lambda^{2}} f\left(a \cos \psi, a \omega_{0} \sin \psi,-a k \sin \psi\right) \sin \psi, \\
& \frac{\partial \varphi}{\partial \psi}=-\frac{\varepsilon}{a \lambda^{2}} f\left(a \cos \psi, a \omega_{0} \sin \psi,-a k \sin \psi\right) \cos \psi
\end{aligned}
$$

The obtained equations (2.31) are equations in the standard form in the Bogolyubov sense.

The right-hand sides of Eqs. (2.31) can be represented as the Fourier series

$$
\begin{aligned}
-\frac{\varepsilon}{\lambda^{2}} f\left(a \cos \psi, a \omega_{0} \sin \psi,-a k \sin \psi\right) \sin \psi & =\varepsilon f_{1}(a, \psi)=\varepsilon \sum_{n} f_{1 n}(a) e^{i n \psi} . \\
-\frac{\varepsilon}{a \lambda^{2}} f\left(a \cos \psi, a \omega_{0} \sin \psi,-a k \sin \psi\right) \cos \psi & =\varepsilon f_{2}(a, \psi)=\varepsilon \sum_{n} f_{2 n}(a) e^{i n \psi}
\end{aligned}
$$

where

$$
f_{1 n}(a)=\frac{1}{2 \pi} \int_{0}^{2 \pi} f_{1}(a, \psi) e^{-i n \psi} d \psi, \quad f_{2 n}(a)=\frac{1}{2 \pi} \int_{0}^{2 \pi} f_{2}(a, \psi) e^{-i n \psi} d \psi
$$


Denote

$$
\tilde{f}_{1}(a, \psi)=\sum_{n \neq 0} \frac{1}{i n} f_{1 n}(a) e^{i n \psi}, \quad \tilde{f}_{2}(a, \psi)=\sum_{n \neq 0} \frac{1}{i n} f_{2 n}(a) e^{i n \psi} .
$$

Changing the variables in (2.31) according to the relations

$$
a=a_{1}+\varepsilon v_{1}\left(a_{1}, \psi_{1}\right), \quad \varphi=\varphi_{1}+\varepsilon w_{1}\left(a_{1}, \psi_{1}\right),
$$

where $\psi_{1}=k t-\omega_{0} t+\varphi_{1}, a_{1}$ and $\varphi_{1}$ are new variables, and the functions $v_{1}\left(a_{1}, \psi_{1}\right)$ and $w_{1}\left(a_{1}, \varphi_{1}\right)$ satisfy the relations

$$
v_{1}\left(a_{1}, \psi_{1}\right)=\tilde{f}_{1}\left(a_{1}, \psi_{1}\right), \quad w_{1}\left(a_{1}, \psi_{1}\right)=\tilde{f}_{2}\left(a_{1}, \psi_{1}\right),
$$

instead of Eqs. (2.31), we obtain the following system of equations in the new variables $a_{1}$ and $\varphi_{1}$ :

$$
\begin{aligned}
& \frac{\partial a_{1}}{\partial \psi}=\varepsilon f_{10}\left(a_{1}\right)+\varepsilon^{2}\left[f_{1 a}^{\prime}\left(a_{1}, \psi_{1}\right) v_{1}\left(a_{1}, \psi_{1}\right)+f_{1 \psi}^{\prime}\left(a_{1}, \psi_{1}\right) w_{1}\left(a_{1}, \psi_{1}\right)\right]+\varepsilon^{3}, \\
& \frac{\partial \varphi_{1}}{\partial \psi}=\varepsilon f_{20}\left(a_{1}\right)+\varepsilon^{2}\left[f_{2 a}^{\prime}\left(a_{1}, \psi_{1}\right) v_{1}\left(a_{1}, \psi_{1}\right)+f_{2 \psi}^{\prime}\left(a_{1}, \varphi_{1}\right) w_{1}\left(a_{1}, \varphi_{1}\right)\right]+\varepsilon^{3} .
\end{aligned}
$$

By neglecting the terms of the second order of smallness with respect to $\varepsilon$ on the right-hand sides of system (2.35), we obtain the averaged system of the first approximation

$$
\frac{\partial a_{1}}{\partial \psi}=\varepsilon f_{10}\left(a_{1}\right), \quad \frac{\partial \varphi_{1}}{\partial \psi}=\varepsilon f_{20}\left(a_{1}\right)
$$

or, in view of the reasoning presented above, the system

$$
\begin{aligned}
& \frac{\partial a_{1}}{\partial t}+\omega_{0}^{\prime} \frac{\partial a_{1}}{\partial x}=\frac{\varepsilon}{2 \pi \omega_{0}} \int_{0}^{2 \pi} f\left(a_{1} \cos \psi_{1}, a_{1} \omega_{0} \sin \psi_{1},-a_{1} k \sin \psi_{1}\right) \sin \psi_{1} d \psi_{1} \\
& \frac{\partial \varphi_{1}}{\partial a}+\omega_{0}^{\prime} \frac{\partial \varphi_{1}}{\partial x}=\frac{\varepsilon}{2 \pi \omega_{0} a_{1}} \int_{0}^{2 \pi} f\left(a_{1} \cos \psi_{1}, a_{1} \omega_{0} \sin \psi_{1},-a_{1} k \sin \psi_{1}\right) \cos \psi_{1} d \psi_{1} .
\end{aligned}
$$

Equations of the first approximation (2.37) can easily be obtained by direct averaging of the right-hand sides of system (2.31) over $\psi$ in view of the variance relation $(2.4)$. Moreover, as expected, Eqs. (2.37) completely coincide with system (2.19).

Note that, by using the described asymptotic method of nonlinear mechanics, one can successfully construct asymptotic approximate solutions of more complicated nonlinear equations similar to the Klein-Gordon equation for small $\varepsilon$. Thus, we can construct solutions of a nonlinear equation with slowly varying parameters of the form

$$
\frac{\partial^{2} u}{\partial t^{2}}-C^{2}(\tau) \frac{\partial^{2} u}{\partial x^{2}}+\lambda^{2}(\tau) u=\varepsilon f\left(\theta, u, \frac{\partial u}{\partial t}, \frac{\partial u}{\partial x}\right),
$$

where $\varepsilon$ is a small positive parameter, $\tau=\varepsilon t$ is slow time, and $d \theta / d t=\nu(\tau)$ both in the resonance and nonresonance case. The obtained relations for both the first and second approximations allow one to study non stationary conditions for a system described by an equation of the form (2.38). 
Furthermore, it is also very interesting to investigate the action of white noise $\xi^{Y}$ with intensity 1 on a system described by a nonlinear equation of the form (2.1). In this case, we consider the equation

$$
\frac{\partial^{2} u}{\partial t^{2}}-C^{2} \frac{\partial^{2} u}{\partial x}+\lambda^{2} u=\varepsilon f\left(u, \frac{\partial u}{\partial t}, \frac{\partial u}{\partial x}\right)+\sqrt{\varepsilon} g\left(u, \frac{\partial u}{\partial t}, \frac{\partial u}{\partial x}\right) \xi^{Y},
$$

for which the problem is reduced to finding a system of stochastic differential equations for the amplitude and phase and to the construction and analysis of the Fokker PlanckKolmogorov equation for the probability density of the amplitude and phase of the system under consideration.

These problems will be studied in one of the subsequent papers.

\section{NOW WE ARE PASSING TO CONSTRUCTION OF ASYMPTOTIC SOLUTION OF PERTURBED BRETHERTON EQUATION}

Consider the equation

$$
u_{t t}+u_{x x x x}+u_{x x}+u=\varepsilon f\left(\nu t, u, u_{t}, u_{x}\right),
$$

which, for $f\left(\nu t, u, u_{t}, u_{x}\right)=u^{3}$, turns into the model Bretherton equation [1]

$$
u_{t t}+u_{x x x x}+u_{x x}+u=\varepsilon u^{3} .
$$

Here,

$$
u_{t t}=\frac{\partial^{2} u}{\partial t^{2}}, \quad u_{x x}=\frac{\partial^{2} u}{\partial x^{2}}, \quad u_{x x x x}=\frac{\partial^{4} u}{\partial x^{3}} .
$$

For $\varepsilon=0$; Eq. (3.1) [as well as Eq. (3.2)] degenerates into a nonperturbed equation

$$
u_{t t}+u_{x x x x}+u_{x x}+u=0
$$

which admits the running-wave solution

$$
u=a \cos \psi, \quad \psi=k x-\omega t+\varphi,
$$

where $a$ and $\varphi$ are constants, and $k$ and $\omega$ satisfy the dispersion relation

$$
\omega^{2}=k^{4}-k^{2}+1 \text {. }
$$

Below, we present the principal scheme of the construction of the first and second approximations of an asymptotic solution according to the basic methods of nonlinear mechanics (the KBM method). This may turn out to be useful for the solution of specific problems of natural sciences that require the investigation of wave processes, taking into account the effect of nonlinear time-dependent perturbing forces, random external perturbations, and other factors.

Thus, passing to the construction of an approximate asymptotic solution of Eq. (3.1), we assume that $\varepsilon>0$ is a small parameter and the function $f\left(\nu t, u, u_{t}, u_{x}\right)$ is periodic (or almost periodic) in $\theta=\nu t$ and differentiable sufficiently many times with respect to the other arguments for all their finite values.

Then, according to the known statements of asymptotic methods of nonlinear mechanics (the KBM method), an asymptotic solution of Eq. (3.1) (for $\varepsilon \rightarrow 0$ ) is sought in the 
form of the following series with slowly varying parameters ${ }^{1}[3,4]$ :

$$
\begin{aligned}
& u=a(t, x) \cos \psi(t, x)+\varepsilon u_{1}(a, \psi)+\varepsilon^{2} u_{2}(a, \psi)+\varepsilon^{3} \ldots, \\
& \psi=k x-\omega t+\varphi(t, x),
\end{aligned}
$$

where the slowly varying parameters $a(t, x)$ and $\varphi(t, x)$ (the amplitude of the first harmonic and the phase) satisfy the system of equations

$$
\begin{aligned}
& \frac{\partial a}{\partial t}=\varepsilon A_{1}(a)+\varepsilon^{2} A_{2}(a)+\varepsilon^{3} \ldots \\
& \frac{\partial a}{\partial x}=\varepsilon B_{1}(a)+\varepsilon^{2} B_{2}(a)+\varepsilon^{3} \ldots \\
& \frac{\partial \varphi}{\partial t}=\varepsilon C_{1}(a)+\varepsilon^{2} C_{2}(a)+\varepsilon^{3} \ldots \\
& \frac{\partial \varphi}{\partial x}=\varepsilon D_{1}(a)+\varepsilon^{2} D_{2}(a)+\varepsilon^{3} \ldots
\end{aligned}
$$

Here and in what follows, we denote

$$
\frac{\partial a}{\partial t}=a_{t}, \quad \frac{\partial a}{\partial x}=a_{x}, \quad \frac{\partial \varphi}{\partial t}=\varphi_{t}, \quad \frac{\partial \varphi}{\partial x}=\varphi_{x}, \quad \frac{\partial^{2} a}{\partial t^{2}}=a_{t t}, \quad \frac{\partial^{2} a}{\partial x^{2}}=a_{x x}, \quad \text { etc. }
$$

It is clear that

$$
\begin{aligned}
& \psi_{t}=-\omega+\varepsilon C_{1}(a)+\varepsilon^{2} C_{2}(a)+\varepsilon^{3} \ldots, \\
& \psi_{x}=k+\varepsilon D_{1}(a)+\varepsilon^{2} D_{2}(a)+\varepsilon^{3} \ldots
\end{aligned}
$$

Differentiating the right-hand sides of Eqs. (3.7), we obtain the following expressions (to within quantities of order $\varepsilon^{2}$ ), which are necessary for further discussion:

$$
\begin{array}{ll}
\left(a_{t}\right)^{2}=\varepsilon^{2} A_{1}^{2}(a)+\varepsilon^{3} \ldots, & a_{t t}=\varepsilon^{2} \frac{d A_{1}(a)}{d a} A_{1}(a)+\varepsilon^{3} \ldots \\
\left(a_{x}\right)^{2}=\varepsilon^{2} B_{1}^{2}(a)+\varepsilon^{3} \ldots, & a_{x x}=\varepsilon^{2} \frac{d B_{1}(a)}{d a} B_{1}(a)+\varepsilon^{3} \ldots \\
\left(\varphi_{t}\right)^{2}=\varepsilon^{2} C_{1}^{2}(a)+\varepsilon^{3} \ldots, & \varphi_{t t}=\varepsilon^{2} \frac{d C_{1}(a)}{d a} A_{1}(a)+\varepsilon^{3} \ldots, \\
\left(\varphi_{x}\right)^{2}=\varepsilon^{2} D_{1}^{2}(a)+\varepsilon^{3} \ldots, & \varphi_{x x}=\varepsilon^{2} \frac{d D_{1}(a)}{d a} B_{1}(a)+\varepsilon^{3} \ldots
\end{array}
$$

Denote

$$
u^{0}(a, \dot{\psi})=a(t, x) \cos \psi(t, x)
$$

By differentiating the terms of series (3.6) with respect to $t$ and $x$, we get

$$
\begin{aligned}
u_{t}^{0}= & a_{t} \cos \psi-a \varphi_{t} \sin \psi+a \omega \sin \psi, \\
u_{t t}^{0}= & 2\left[a_{t} \omega \sin \psi+a \varphi_{t} \omega \cos \psi\right] \\
& +a_{t t} \cos \psi-a \varphi_{t t} \sin \psi-2 a_{t} \varphi_{t} \sin \psi-a \varphi_{t}^{2} \cos \psi-a \omega^{2} \cos \psi,
\end{aligned}
$$

\footnotetext{
1 Below, for simplicity, we assume that the right-hand side of Eq. (3.1) does not depend on $\nu t$
} 


$$
\begin{aligned}
u_{x}^{0}= & a_{x} \cos \psi-a \varphi_{x} \sin \psi-a k \sin \psi \\
u_{x x}^{0}= & -2\left[a_{x} k \sin \psi+a \varphi_{x} k \cos \psi\right] \\
& +a_{x x} \cos \psi-a \varphi_{x x} \sin \psi-2 a_{x} \varphi_{x} \sin \psi-a \varphi_{x}^{2} \cos \psi-a k^{2} \cos \psi, \\
u_{x x x x}^{0}= & 4\left[a_{x} k^{3} \sin \psi+a \varphi_{x} k^{3} \cos \psi\right]+6\left[2 a_{x} \varphi_{x} k+a \varphi_{x x} k^{2}\right] \sin \psi \\
& -6\left[a_{x x} k^{2}-a \varphi_{x}^{2} k^{2}\right] \cos \psi+\varepsilon^{3} \cdots+a k^{4} \cos \psi
\end{aligned}
$$

Further, we determine the derivatives of $u_{1}(a, \psi)$ with respect to $t$ and $x$ :

$$
\begin{aligned}
u_{1 t}(a, \psi) & =u_{1 a}(a, \psi) a_{t}+u_{1 \psi}(a, \psi) \varphi_{t}-u_{1 \psi}(a, \psi) \omega \\
u_{1 t t}(a, \psi) & =-2\left[\omega a_{t} u_{1 \psi a}(a, \psi)+\omega \varphi_{t} u_{1 \psi \psi}(a, \psi)\right]+\omega^{2} u_{1 \psi \psi}(a, \psi)+\varepsilon^{2} \ldots \\
u_{1 x}(a, \psi) & =a_{x} u_{1 a}(a, \psi)+\varphi_{x} u_{1 \psi}(a, \psi)+k u_{1 \psi}(a, \psi) \\
u_{1 x x}(a, \psi) & =2\left[k a_{x} u_{1 a \psi}(a, \psi)+k \varphi_{x} u_{1 \psi \psi}(a, \psi)\right]+k^{2} u_{1 \psi \psi}(a, \psi)+\varepsilon^{3} \ldots \\
u_{1 x x x x}(a, \psi) & =4\left[k^{3} a_{x} u_{1 a \psi \psi \psi}(a, \psi)+k^{3} \varphi_{x} u_{1 \psi \psi \psi}(a, \psi)\right]+k^{4} u_{1 \psi \psi \psi}(a, \psi)+\varepsilon^{3}
\end{aligned}
$$

For the term $u_{2}(a, \psi)$, to within quantities of the second order inclusive with respect to $\varepsilon$, we get

$$
\begin{gathered}
u_{2 t}(a, \psi)=-u_{2 \psi}(a, \psi) \omega+\varepsilon^{3} \ldots, \quad u_{2 t t}(a, \psi)=u_{2 \psi \psi}(a, \psi) \omega^{2} \\
u_{2 x}(a, \psi)=u_{2 \psi}(a, \psi) k+\varepsilon^{3} \ldots, \quad u_{2 x x}(a, \psi)=u_{2 \psi \psi}(a, \psi) k^{2} \\
u_{2 x x x x}(a, \psi)=u_{2 \psi \psi \psi \psi}(a, \psi) k^{4}
\end{gathered}
$$

By substituting expressions (3.6), (3.10), (3.12), (3.14), (3.15), (3.17), and (3.19)-(3.23) for $u, u_{t t}, u_{x x}$, and $u_{x x x x}$ on the left-hand side of Eq. (3.1) and taking relations (3.9) into account, to within quantities of order $\varepsilon^{2}$, we obtain

$$
\begin{aligned}
u_{t t}+ & u_{x x x x}+u_{x x}+u=-\omega^{2} a \cos \psi+k^{4} a \cos \psi-k^{2} a \cos \psi+a \cos \psi \\
& +\varepsilon\left\{\left[k^{4} u_{1 \psi \psi \psi} \psi(a, \psi)+\omega^{2} u_{1 \psi \psi}(a, \psi)+k^{2} u_{1 \psi \psi}(a, \psi)+u_{1}(a, \psi)\right]\right. \\
& \left.+2\left[A_{1}(a) \omega+\left(2 k^{3}-k\right) B_{1}(a)\right] \sin \psi+2\left[C_{1}(a) \omega+\left(2 k^{3}-k\right) D_{1}(a)\right] a \cos \psi\right\} \\
& +\varepsilon^{2}\left\{\left[k^{4} u_{2 \psi \psi \psi \psi}(a, \psi)+\omega^{2} u_{2 \psi \psi}(a, \psi)+k^{2} u_{2 \psi \psi}(a, \psi)+u_{2}(a, \psi)\right]\right. \\
& +2\left[A_{2}(a) \omega+\left(2 k^{3}-k\right) B_{2}(a)\right] \sin \psi+2\left[C_{2}(a) \omega+\left(2 k^{3}-k\right) D_{2}(a)\right] a \cos \psi \\
& +\left[-a \frac{d C_{1}(a)}{d a} A_{1}(a)-2 A_{1}(a) C_{1}(a)+6\left(2 k^{2} B_{1}(a) D_{1}(a)\right.\right. \\
& \left.\left.+a k^{2} \frac{d D_{1}(a)}{d a} B_{1}(a)\right)-a \frac{d D_{1}(a)}{d a} B_{1}(a)-2 B_{1}(a) D_{1}(a)\right] \sin \psi \\
& +\left[\frac{d A_{1}(a)}{d a} A_{1}(a)-a C_{1}^{2}(a)-6\left(\frac{d B_{1}(a)}{d a} B_{1}(a) k^{2}-a D_{1}^{2}(a) k^{2}\right)\right. \\
& \left.+\frac{d B_{1}(a)}{d a} B_{1}(a)-a D_{1}^{2}(a)\right] \cos \psi+4\left[B_{1}(a) k^{3} u_{1 a \psi \psi \psi}(a, \psi)\right.
\end{aligned}
$$




$$
\begin{aligned}
& \left.+D_{1}(a) k^{3} u_{1 \psi \psi \psi \psi}(a, \psi)\right]-2\left[\omega A_{1}(a) u_{1 a \psi}(a, \psi)+\omega C_{1}(a) u_{1 \psi \psi}(a, \psi)\right] \\
& \left.+2\left[B_{1}(a) k u_{1 a \psi}(a, \psi)+D_{1}(a) k u_{1 \psi \psi}(a, \psi)\right]\right\}+\varepsilon^{3} \ldots
\end{aligned}
$$

By substituting expressions (3.6), (3.10), (3.14), (3.16), and (3.18) for $u(a, \psi), u_{t}(a, \psi)$, and $u_{x}(a, \psi)$ on the right-hand side of Eq. (3.1) and expanding the result in the Taylor series, we get

$$
\begin{aligned}
& \varepsilon f\left(u, u_{t}, u_{x}\right)=\varepsilon f(a \cos \psi, a \omega \sin \psi,-a k \sin \psi) \\
& +\varepsilon^{2}\left\{f_{u}^{\prime}(a \cos \psi, a \omega \sin \psi,-a k \sin \psi) u_{1}(a, \psi)\right. \\
& +f_{u_{t}}^{\prime}(a \cos \psi, a \omega \sin \psi,-a k \sin \psi)\left(A_{1}(a) \cos \psi-a C_{1}(a) \sin \psi-\omega u_{1 \psi}(a, \psi)\right) \\
& \left.+f_{u_{x}}^{\prime}(a \cos \psi, a \omega \sin \psi,-a k \sin \psi)\left(B_{1}(a) \cos \psi-a D_{1}(a) \sin \psi+k u_{1 \psi}(a, \psi)\right)\right\}+\varepsilon^{3} \ldots
\end{aligned}
$$

By equating the coefficients of the same powers of $\varepsilon$ in expressions (3.24) and (3.25), we obtain the following chain of equations for the determination of the functions $u_{i}(a, \psi)$, $A_{i}(a), B_{i}(a), C_{i}(a)$, and $D_{i}(a), i=1,2,3, \ldots$ :

$$
\begin{aligned}
& \varepsilon^{0}:-\omega^{2} a \cos \psi+a k^{4} \cos \psi-a k^{2} \cos \psi+a \cos \psi=0, \\
& \varepsilon^{1}: k^{4} u_{1 \psi \psi \psi \psi}(a, \psi)+\omega^{2} u_{1 \psi \psi}(a, \psi)+k^{2} u_{1 \psi \psi}(a, \psi)+u_{1}(a, \psi) \\
& \quad+2\left[A_{1}(a) \omega+2\left(k^{3}-k\right) B_{1}(a)\right] \sin \psi \\
& \quad+2\left[C_{1}(a) \omega+\left(2 k^{3}-k\right) D_{1}(a)\right] a \cos \psi \\
& =\varepsilon f(a \cos \psi, a \omega \sin \psi,-a k \sin \psi),
\end{aligned}
$$

$$
\begin{aligned}
\varepsilon^{2}: k^{4} & u_{2 \psi \psi \psi \psi}(a, \psi)+\omega^{2} u_{2 \psi \psi}(a, \psi)+k^{2} u_{2 \psi \psi}(a, \psi)+u_{2}(a, \psi) \\
+ & 2\left[A_{2}(a) \omega+\left(2 k^{3}-k\right) B_{2}(a)\right] \sin \psi+2\left[C_{2}(a) \omega+\left(2 k^{3}-k\right) D_{2}(a)\right] a \cos \psi \\
+ & {\left[-a \frac{d C_{1}(a)}{d a} A_{1}(a)-2 A_{1}(a) C_{1}(a)+6\left(2 k^{2} B_{1}(a) D_{1}(a)\right.\right.} \\
+ & \left.\left.a k^{2} \frac{d D_{1}(a)}{d a} B_{1}(a)\right)-a \frac{d D_{1}(a)}{d a} B_{1}(a)-2 B_{1}(a) D_{1}(a)\right] \sin \psi \\
+ & {\left[\frac{d A_{1}(a)}{d a} A_{1}(a)-a C_{1}^{2}(a)-6\left(\frac{d B_{1}(a)}{d a} B_{1}(a) k^{2}-a D_{1}^{2}(a) k^{2}\right.\right.} \\
+ & \left.\frac{d B_{1}(a)}{d a} B_{1}(a)-a D_{1}^{2}(a)\right] \cos \psi \\
+ & 4\left[B_{1}(a) k^{3} u_{1 a \psi \psi \psi}(a, \psi)+D_{1}(a) k^{3} u_{1 \psi \psi} \psi \psi(a, \psi)\right] \\
& -2\left[\omega A_{1}(a) u_{1 a \psi}(a, \psi)+\omega C_{1}(a) u_{1 \psi \psi}(a, \psi)\right] \\
+ & 2\left[B_{1}(a) k u_{1 a \psi}(a, \psi)+D_{1}(a) k u_{1 \psi \psi}(a, \psi)\right] \\
& =f_{u}^{\prime}(a \cos \psi, a \omega \sin \psi,-a k \sin \psi) u_{1}(a, \psi) \\
+ & f_{u_{t}}^{\prime}(a \cos \psi, a \omega \sin \psi,-a k \sin \psi)\left(A_{1}(a) \cos \psi-a C_{1}(a) \sin \psi-\omega u_{1 \psi}(a, \psi)\right) \\
+ & f_{u_{x}}^{\prime}(a \cos \psi, a \omega \sin \psi,-a k \sin \psi)\left(B_{1}(a) \cos \psi-a D_{1}(a) \sin \psi+k u_{1 \psi}(a, \psi)\right),
\end{aligned}
$$


Equations (3.26)-(3.28) can be rewritten in the form

$$
\begin{aligned}
& -\omega^{2} a \cos \psi+a k^{4} \cos \psi-a k^{2} \cos \psi+a \cos \psi=0, \\
& k^{4} u_{1 \psi \psi \psi} \psi(a, \psi)+\left(\omega^{2}+k^{2}\right) u_{1 \psi \psi}(a, \psi)+u_{1}(a, \psi)=f_{0}(a, \psi) \\
& -2\left[A_{1}(a) \omega+\left(2 k^{3}-k\right) B_{1}(a)\right] \sin \psi-2\left[C_{1}(a) \omega+\left(2 k^{3}-k\right) D_{1}(a)\right] a \cos \psi,
\end{aligned}
$$

$$
\begin{aligned}
k^{4} & u_{2 \psi \psi \psi \psi}(a, \psi)+\left(\omega^{2}+k^{2}\right) u_{2 \psi \psi}(a, \psi)+u_{2}(a, \psi) \\
= & f_{1}(a, \psi)-\left\{2\left[A_{2}(a) \omega+\left(2 k^{3}-k\right) B_{2}(a)\right]-a \frac{d C_{1}(a)}{d a} A_{1}(a)-2 A_{1}(a) C_{1}(a)\right. \\
& \left.+6\left(2 k^{2} B_{1}(a) D_{1}(a)+a k^{2} \frac{d D_{1}(a)}{d a} B_{1}(a)\right)-a \frac{d D_{1}(a)}{d a} B_{1}(a)-2 B_{1}(a) D_{1}(a)\right\} \sin \psi \\
& -\left\{2\left[C_{2}(a) \omega+\left(2 k^{3}-k\right) D_{2}(\omega)\right] a+\frac{d A_{1}(a)}{d a} A_{1}(a)-a C_{1}^{2}(a)\right. \\
& \left.-6\left(\frac{d B_{1}(a)}{d a} B_{1}(a) k^{2}-a D_{1}^{2}(a) k^{2}\right)+\frac{d B_{1}(a)}{d a} B_{1}(a)-a D_{1}^{2}(a)\right\} \cos \psi
\end{aligned}
$$

where, for simplicity, we have introduced the following notation:

$$
\begin{aligned}
& f_{0}(a, \psi)=f(a \cos \psi, a \omega \sin \psi,-a k \sin \psi), \\
& f_{1}(a, \psi)=f_{u}^{\prime}(a \cos \psi, a \omega \sin \psi,-a k \sin \psi) u_{1}(a, \psi) \\
& \quad+f_{u_{t}}^{\prime}(a \cos \psi, a \omega \sin \psi,-a k \sin \psi)\left(A_{1}(a) \cos \psi-a C_{1}(a) \sin \psi-\omega u_{1 \psi}(a, \psi)\right) \\
& \quad+f_{u_{x}}^{\prime}(a \cos \psi, a \omega \sin \psi,-a k \sin \psi)\left(B_{1}(a) \cos \psi-a D_{1}(a) \sin \psi+k u_{1 \psi}(a, \psi)\right) \\
& \quad-4\left[B_{1}(a) k^{3} u_{1 a \psi \psi \psi}(a, \psi)+D_{1}(a) k^{3} u_{1 \psi \psi \psi} \psi(a, \psi)\right] \\
& \quad+2\left[\omega A_{1}(a) u_{1 a \psi}(a, \psi)+\omega C_{1}(a) u_{1 \psi \psi}(a, \psi)\right] \\
& \quad-2\left[B_{1}(a) k u_{1 a \psi}(a, \psi)+D_{1}(a) k u_{1 \psi \psi}(a, \psi)\right] .
\end{aligned}
$$

By virtue of the dispersion relation (3.5), Eq. (3.29) is identically equal to zero.

To determine $u_{1}(a, \psi), A_{1}(a), B_{1}(a), C_{1}(a)$, and $D_{1}(a)$ (i.e., $\frac{\partial a}{\partial t}, \frac{\partial a}{\partial x}, \frac{\partial \varphi}{\partial t}$, and $\frac{\partial \varphi}{\partial x}$ in the first approxi mation) from Eq. (3.30), we consider the Fourier expansion of the function $f_{0}(a, \psi)$, which is obviously periodic in $\psi$ (recall that $\left.\psi=k x-\omega t+\varphi\right)$. We have

$$
f_{0}(a, \psi)=g_{0}(a)+\sum_{n=1}^{\infty}\left[g_{n}(a) \cos n \psi+h_{n}(a) \sin n \psi\right],
$$

where

$$
\begin{aligned}
& g_{n}(a)=\frac{1}{\pi} \int_{0}^{2 \pi} f_{0}(a, \psi) \cos n \psi d \psi, \\
& h_{n}(a)=\frac{1}{\pi} \int_{0}^{2 \pi} f_{0}(a, \psi) \sin n \psi d \psi, \quad n=0,1,2, \ldots
\end{aligned}
$$


We seek the function $u_{1}(a, \psi)$ in the form of a periodic function of $\psi$, namely,

$$
u_{1}(a, \psi)=v_{0}(a) \sum_{n=1}^{\infty}\left[v_{n}(a) \cos n \psi+w_{n}(a) \sin n \psi\right] .
$$

By substituting the right-hand sides of expressions (3.34) and (3.36) into Eq. (3.30), we obtain the expression

$$
\begin{aligned}
& v_{0}(a)+\sum_{n=1}^{\infty}\left[k^{4} n^{4}-\left(\omega^{2}+k^{2}\right) n^{2}+1\right]\left[v_{n}(a) \cos n \psi+w_{n}(a) \sin n \psi\right] \\
& =g_{0}(a)+\sum_{n=2}^{\infty}\left[g_{n}(a) \cos n \psi+h_{n}(a) \sin n \psi\right] \\
& \quad+\left\{g_{1}(a)-2 a\left[C_{1}(a) \omega+\left(2 k^{3}-k\right) D_{1}(a)\right]\right\} \cos \psi \\
& \quad+\left\{h_{1}(a)-2\left[A_{1}(a) \omega+\left(2 k^{3}-k\right) B_{1}(a)\right]\right\} \sin \psi,
\end{aligned}
$$

whence, by equating the coefficients of the same harmonics, we get $\left(\omega^{2}=k^{4}-k^{2}+1\right)$

$$
\begin{gathered}
C_{1}(a) \omega+\left(2 k^{3}-k\right) D_{1}(a)=\frac{g_{1}(a)}{2 a}, \quad A_{1}(a) \omega+\left(2 k^{3}-k\right) B_{1}(a)=\frac{h_{1}(a)}{a}, \\
v_{0}(a)=g_{0}(a), \quad v_{n}(a)=\frac{g_{n}(a)}{k^{4} n^{4}-\left(\omega^{2}+k^{2}\right) n^{2}+1}, \\
w_{n}(a)=\frac{h_{n}(a)}{k^{4} n^{4}-\left(\omega^{2}+k^{2}\right) n^{2}+1}, \quad n=2,3, \ldots
\end{gathered}
$$

Equalities (3.38) guarantee that the function $u_{1}(a, \psi)$ has no secular terms.

Then the required function $u_{1}(a, \psi)$ can be represented as follows:

$$
u_{1}(a, \psi)=g_{0}(a)+\sum_{n=2}^{\infty} \frac{1}{k^{4} n^{4}-\left(\omega^{2}+k^{2}\right) n^{2}+1}\left(g_{n}(a) \cos n \psi+h_{n}(a) \sin n \psi\right),
$$

where $g_{n}(a) h_{n}(a), n=0,1,2,3, \ldots$, are defined by (3.35).

Taking the system of equations (3.7) into account, we can represent Eqs. (3.38) to within quantities of the first order of smallness in the form

$$
\begin{aligned}
& \frac{\partial a}{\partial t}+\frac{2 k^{3}-k}{\omega} \frac{\partial a}{\partial x}=\frac{\varepsilon}{2 \pi \omega} \int_{0}^{2 \pi} f_{0}(a, \psi) \sin \psi d \psi, \\
& \frac{\partial \varphi}{\partial t}+\frac{2 k^{3}-k}{\omega} \frac{\partial \varphi}{\partial x}=\frac{\varepsilon}{2 \pi a \omega} \int_{0}^{2 \pi} f_{0}(a, \psi) \cos \psi d \psi
\end{aligned}
$$

For the construction of the second approximation, it is necessary to find $u_{2}(a, \psi)$, $\dot{A}_{2}(a, \psi), B_{2}(a, \psi), C_{2}(a, \psi)$, and $D_{2}(a, \psi)$ by using Eq. (3.28) or (3.31). By expanding the function $f_{1}(a, \psi)$ in a Fourier series, we get

$$
f_{1}(a, \psi)=g_{0}^{(1)}(a)+\sum_{n=1}^{\infty}\left[g_{n}^{(1)}(a) \cos n \psi+h_{n}^{(1)}(a) \sin n \psi\right]
$$


where

$$
\begin{aligned}
& g_{n}^{(1)}(a)=\frac{1}{\pi} \int_{0}^{2 \pi} f_{1}(a, \psi) \cos n \psi d \psi, \\
& h_{n}^{(1)}(a)=\frac{1}{\pi} \int_{0}^{2 \pi} f_{1}(a, \psi) \sin n \psi d \psi, \quad n=0,1,2, \ldots
\end{aligned}
$$

We seek $u_{2}(a, \psi)$ in the form of a series

$$
u_{2}(a, \psi)=v_{0}^{(1)}(a)+\sum_{n=1}^{\infty}\left[v_{n}^{(1)}(a) \cos n \psi+h_{n}^{(1)}(a) \sin n \psi\right],
$$

substituting it in Eq. (3.31); the unknown coefficients $v_{n}^{(1)}(a), w_{n}^{(1)}(a), n=0,1,2, \ldots$, of harmonics can be determined as in the previous case. By substituting expressions (3.44) for $u_{2}(a, \psi)$ and $(3.42)$ for $f_{1}(a, \psi)$ on the left-hand and right-hand sides of Eq. (3.31) and equating the coefficients of the same harmonics, we obtain

$$
\begin{aligned}
& v_{0}^{(1)}(a)=g_{0}^{(1)}(a), \quad v_{n}^{(1)}(a)=\frac{g_{n}^{(1)}(a)}{k^{4} n^{4}-\left(\omega^{2}+k^{2}\right) n^{2}+1}, \\
& w_{n}^{(1)}(a)=\frac{h_{n}^{(1)}(a)}{k^{4} n^{4}-\left(\omega^{2}+k^{2}\right) n^{2}+1}, \quad n=2,3,4, \ldots,
\end{aligned}
$$

and

$$
\begin{aligned}
& 2\left[C_{2}(a) \omega+\left(2 k^{3}-k\right) D_{2}(a)\right] a+\left[\frac{d A_{1}(a)}{d a} A_{1}(a)-a C_{1}^{2}(a)\right. \\
& \left.\quad-6\left(\frac{d B_{1}}{d a} B_{1}(a) k^{2}-a D_{1}^{2}(a) k^{2}\right)+\frac{d B_{1}(a)}{d a} B_{1}(a)-a D_{1}^{2}(a)\right]=g_{1}^{(1)}(a), \\
& 2\left[A_{2}(a) \omega+\left(2 k^{3}-k\right) B_{2}(a)\right]-a \frac{d C_{1}(a)}{d a} A_{1}(a)-2 A_{1}(a) C_{1}(a) \\
& \quad+6\left(2 k^{2} B_{1}(a) D_{1}(a)+a k^{2} \frac{d D_{1}(a)}{d a} B_{1}(a)\right)-\frac{d D_{1}(a)}{d a} a B_{1}(a)-2 B_{1}(a) D_{1}(a)=h_{1}^{(1)}(a) .
\end{aligned}
$$

We can now write the following expression for $u_{2}(a, \psi)$ :

$$
u_{2}(a, \psi)=g_{0}^{(1)}(a)+\sum_{n=2}^{\infty} \frac{1}{k^{4} n^{4}-\left(\omega^{2}+k^{2}\right) n^{2}+1}\left[g_{n}^{(1)}(a) \cos n \psi+h_{n}^{(1)}(a) \sin n \psi\right]
$$

where $g_{n}^{(1)}(a)$ and $h_{n}^{(1)}(a), n=0,2,3, \ldots$ are defined by (3.43). Expressions (3.46) guarantee the absence of secular terms in (3.47).

According to (3.46), we obtain expressions for $A_{2}(a), B_{2}(a), C_{2}(a)$, and $D_{2}(a)$ that are necessary for the construction of Eqs. (3.7) in the second approximation: 


$$
\begin{aligned}
C_{2}(a) \omega & +\left(2 k^{3}-k\right) D_{2}(a)=\frac{g_{1}^{(1)}(a)}{2 a}-\frac{1}{2 a}\left[\frac{d A_{1}(a)}{d a} A_{1}(a)-a C_{1}^{2}(a)\right. \\
& \left.-6\left(\frac{d B_{1}(a)}{d a} B_{a}(a) k^{2}-D_{1}^{2}(a) k^{2}\right)+\frac{d B_{1}(a)}{d a} B_{1}(a)-a D_{1}^{2} 3(a)\right] \\
A_{2}(a) \omega+ & \left(2 k^{3}-k\right) B_{2}(a)=\frac{h_{1}^{(1)}(a)}{2}+\frac{1}{2}\left[\frac{d C_{1}(a)}{d a} A_{1}(a)+2 A_{1}(a) C_{1}(a)\right. \\
& \left.-6\left(2 k^{2} B_{1}(a) D_{1}(a)+a k^{2} \frac{d D_{1}(a)}{d a} B_{1}(a)\right)+\frac{d D_{1}(a)}{d a} a B_{1}(a)+2 B_{1}(a) D_{1}(a)\right] .
\end{aligned}
$$

Taking into account that

$$
\begin{aligned}
& g_{1}^{(1)}(a)=\frac{1}{\pi} \int_{0}^{2 \pi} f_{1}(a, \psi) \cos \psi d \psi, \\
& h_{1 . .}^{(1)}(a)=\frac{1}{\pi} \int_{0}^{2 \pi} f_{1}(a, \psi) \sin \psi d \psi,
\end{aligned}
$$

adding the corresponding equalities in (3.38) and (3.48) together, and using Eqs. (3.7) (and the corresponding coefficients of $\varepsilon$ and $\varepsilon^{2}$ ), we obtain Eqs. (3.41) in the sccond approximation in the form

$$
\begin{aligned}
\frac{\partial a}{\partial t}+\omega^{\prime} \frac{\partial a}{\partial x}= & \frac{1}{2 \pi \omega} \int_{0}^{2 \pi}\left[\varepsilon f_{0}(a, \psi)+\varepsilon^{2} f_{1}(a, \psi)\right] \sin \psi d \psi \\
& +\frac{\varepsilon}{2 \omega}\left\{a \frac{d C_{1}(a)}{d a} A_{1}(a)+2 A_{1}(a) C_{1}(a)\right. \\
& -6\left(2 k^{2} B_{1}(a) D_{1}(a)+a k^{2} \frac{d D_{1}(a)}{d a} B_{1}(a)\right) \\
& \left.+\frac{d D_{1}(a)}{d a} a B_{1}(a)+2 B_{1}(a) D_{1}(a)\right\} \\
\frac{\partial \varphi}{\partial t}+\omega^{\prime} \frac{\partial \varphi}{\partial x}= & \frac{1}{2 \pi \omega a} \int_{0}^{2 \pi}\left[\varepsilon f_{0}(a, \psi)+\varepsilon^{2} f_{1}(a, \psi)\right] \cos \psi d \psi \\
& -\frac{\varepsilon^{2}}{2 \omega a}\left\{\frac{d A_{1}(a)}{d a} A_{1}(a)-C_{1}^{2}(a) a\right. \\
& \left.-6\left(\frac{d B_{1}(a)}{d a} B_{1}(a) k^{2}-a D_{1}^{2}(a) k^{2}\right)+\frac{d B_{1}(a)}{d a} B_{1}(a)-a D_{1}^{2}(a)\right\}
\end{aligned}
$$

where $\omega^{\prime}=\frac{2 k^{3}-k}{\omega}$ is the group velocity [for the linear equation $\left.(3.3)\right]$ and $f_{0}(a, \psi)$ and $f_{1}(a, \psi)$ are defined by (3.32) and (3.33).

Thus, in the first approximation, the equation

$$
u_{t t}+u_{x x x x}+u_{x x}+u=\varepsilon f\left(u, u_{t}, u_{x}\right)
$$


has an asymptotic solution

$$
u(t, x)=a(t, x) \cos \psi(t, x),
$$

where $\psi(t, x)=k x-\omega t+\varphi(t, x)$, and the slowly varying amplitude $a(t, x)$ and phase $\varphi(t, x)$ are to be determined from the system of the first approximation

$$
\begin{aligned}
& \frac{\partial a}{\partial t}+\omega^{\prime} \frac{\partial a}{\partial x}=\frac{\varepsilon}{2 \pi \omega} \int_{0}^{2 \pi} f_{0}(a, \psi) \sin \psi d \psi, \\
& \frac{\partial \varphi}{\partial t}+\omega^{\prime} \frac{\partial \varphi}{\partial x}=\frac{\varepsilon}{2 \pi \omega a} \int_{0}^{2 \pi} f_{0}(a, \psi) \cos \psi d \psi,
\end{aligned}
$$

where $\omega^{2}=k^{4}-k^{2}+1$ is the dispersion relation, $\omega^{\prime}=\frac{2 k^{3}-k}{\omega}$ is the group velocity [both for the linear equation $(3.3)]$, and $f_{0}(a, \psi)$ is defined by $(3.32)$.

As is customary in nonlinear mechanics, an improved first approximation is defined by the expression

$$
u(t, x)=a(t, x) \cos \psi(t, x)+\varepsilon u_{1}(a, \psi),
$$

where $\varepsilon u_{1}(a, \psi)$ is calculated according to formula (3.40), and the slowly varying amplitude $a(t, x)$ and phase $\varphi(t, x)$ are determined from the equations of the first approximation (3.53).

As usual, to obtain the second approximation, it is necessary to substitute the slowly varying amplitude $a(t, x)$ and phase $\varphi(t, x)$ determined from the equations of the second approximation (3.50) into expression (3.54).

Consider the first approximation in more detail.

In order that $u_{1}(a, \psi)$ do not contain secular terms, it is necessary that the functions $A_{1}(a), B_{1}(a), C_{1}(a)$, and $D_{1}(a)$ in Eqs. (3.7) satisfy Eqs. (3.38):

$$
\begin{aligned}
& A_{1}(a) \omega+\left(2 k^{3}-k\right) B_{1}(a)=\frac{h_{1}(a)}{2}, \\
& C_{1}(a) \omega+\left(2 k^{3}-k\right) D_{1}(a)=\frac{g_{1}(a)}{2 a},
\end{aligned}
$$

where $h_{1}(a)$ and $g_{1}(a)$ are defined by $(3.35)$.

We have

$$
\frac{\partial^{2} \varphi}{\partial t \partial x}=\frac{\partial^{2} \varphi}{\partial x \partial t}, \quad \frac{\partial^{2} a}{\partial t \partial x}=\frac{\partial^{2} a}{\partial x \partial t} .
$$

Therefore, according to Eqs. (3.7), we can write (in the first approximation)

$$
A_{1}(a) \frac{d D_{1}(a)}{d a}=B_{1}(a) \frac{d C_{1}(a)}{d a}, \quad A_{1}(a) \frac{d B_{1}(a)}{d a}=B_{1}(a) \frac{d A_{1}(a)}{d a} .
$$

Equations (3.38) and (3.55) give four relations for four unknown functions $A_{1}(a)$, $B_{1}(a), C_{1}(a)$, and $D_{1}(a)$. Having solved these equations and found, in the first approximation, the right-hand sides of Eqs. (3.7) that determine the slowly varying amplitude $a(t, x)$ and phase $\varphi(t, x)$, according to relation (3.40), we can determine $u_{1}(a, \psi)$, which, as indicated above, does not contain secular terms. Note that $C_{1}(a)=\frac{\partial \varphi}{\partial t}$ can be interpreted 
as a "shift of the frequency $\omega$ " (in the first approximation), whereas $D_{1}(a)=\frac{\partial \varphi}{\partial x}$ can be interpreted as a "shift of the wave num ber $k . "$

Nevertheless, in the general case, it is impossible to completely solve the system of equations (3.38) and (3.55) without certain restrictions on the physical problem.

For example, assume that $u(t, x)$ oscillates according to the sine law for all $t$. In this case, we can set $\varepsilon C_{1}(a)=\varphi_{t}(t, x)=0$ and $\varepsilon A_{1}(a)=a_{t}(t, x)=0$. Then we have

$$
D_{1}(a)=\frac{1}{2\left(2 k^{3}-1\right)} g_{1}(a)
$$

as a "shift of the wave number $k "\left[\varepsilon D_{1}(a)=\varphi_{k}(t, x)\right]$, and

$$
B_{1}(a)=\frac{1}{2\left(2 k^{3}-1\right)} h_{1}(a) .
$$

Otherwise, if we assume that, for all $x$, we have a purely sinusoidal wave, then we can set $\varepsilon B_{1}(a)=a_{x}(t, x)=0$ and $\varepsilon D_{1}(a)=\varphi_{t}(t, x)=0$ and solve Eq. (3.38) with respect to $\varepsilon A_{1}(a)=a_{t}(t, x)$ and $\varepsilon C_{1}(a)=\varphi_{t}(t, x)$ to obtain a correction for the amplitude and the shift of the frequency.

Let us consider an example that illustrates the effect of external perturbation on the dispersion relation established for a linear nonperturbed equation.

As such an example, we consider the model Bretherton equation (3.2). holds.

For $\varepsilon=0$, a solution of Eq. (3.2) has the form (3.4), and the dispersion relation (3.5)

Assume that $A_{1}(a)$ and $C_{1}(a)$ are equal to zero. Then, in the first approximation, according to Eqs. (3.38), we have

$$
\left(2 k^{3}-k\right) B_{1}(a)=\frac{1}{2} h_{1}(a), \quad\left(2 k^{3}-k\right) D_{1}(a)=\frac{1}{2 a} g_{1}(a)
$$

or

$$
\begin{aligned}
& \frac{\partial a}{\partial x}=\frac{1}{2 \pi\left(2 k^{3}-k\right)} \int_{0}^{2 \pi} \varepsilon f_{0}(a \cos \psi) \sin \psi d \psi \\
& \frac{\partial \varphi}{\partial x}=\frac{1}{2 \pi a\left(2 k^{3}-k\right)} \int_{0}^{2 \pi} \varepsilon f_{0}(a \cos \psi) \cos \psi d \psi
\end{aligned}
$$

The right-hand side of Eq. (3.2) is $\varepsilon u^{3}$ and, consequently, we should replace $\varepsilon f_{0}$ by $\varepsilon a^{3} \cos ^{3} \psi$ in Eqs. (3.59). Then Eqs. (3.59) take the form

$$
\frac{\partial a}{\partial x}=0, \quad \frac{\partial \varphi}{\partial x}=\frac{3 \varepsilon a^{2}}{8\left(2 k^{3}-k\right)}
$$

Let us determine how the "shift of the wave number" $k$ affects the dispersion relation (3.5). For this purpose, in the dispersion relation (3.5) obtained for the nonperturbed 
equation, we should replace the wave number $k$ by $k+\varphi_{x}$. As a result, we obtain

$$
\begin{aligned}
\omega^{2} & =\left(k+\frac{3 \varepsilon a^{2}}{8\left(2 k^{3}-k\right)}\right)^{4}-\left(k+\frac{3 \varepsilon a^{2}}{8\left(2 k^{3}-k\right)}\right)^{2}+1 \\
& =k^{4}-k^{2}+\frac{3 \varepsilon a^{2}}{4}+1+\varepsilon^{3} \ldots
\end{aligned}
$$

Thus, in the first approximation (to within quantities of order $\varepsilon$ ), the dispersion relation for the model Bretherton equation has the following form:

$$
\omega^{2}=k^{4}-k^{2}+1+\frac{3 \varepsilon a^{2}}{4} .
$$

To obtain an improved first approximation, it is necessary to determine $\varepsilon u_{1}(a, \psi)$. After simple calculations with the use of relations (3.40) and (3.35), we get

$$
\varepsilon u_{1}(a, \psi)=\frac{\varepsilon a^{3}}{32\left(9 k^{4}-1\right)} \cos 3 \psi
$$

and, consequently, in the improved first approximation (under the assumptions made above), a solution of the Bretherton equation has the form

$$
u(t, x)=a \cos \psi+\frac{\varepsilon a^{3}}{32\left(9 k^{4}-1\right)} \cos 3 \psi,
$$

where

$$
\psi=\left(k+\frac{3 \varepsilon a^{3}}{8\left(2 k^{3}-k\right)}\right) x-\omega t+\varphi .
$$

Let us now calculate the corresponding corrections for the frequency and wave number in the second approximation. Since, by assumption, $A_{1}(a)=0, B_{1}(a)=0$, and $C_{1}(a)=0$, in the second approximation, the system of equations (3.50), which defines the slowly varying amplitude $a(t, x)$ and phase $\varphi(t, x)$, takes the form

$$
\begin{aligned}
& \frac{\partial a}{\partial t}+\omega^{\prime} \frac{\partial a}{\partial x}=\frac{1}{2 \pi \omega} \int_{0}^{2 \pi}\left[\varepsilon f_{0}(a, \psi)+\varepsilon^{2} f_{1}(a, \psi)\right] \sin \psi d \psi, \\
& \frac{\partial \varphi}{\partial t}+\omega^{\prime} \frac{\partial \varphi}{\partial x}=\frac{1}{2 \pi \omega a} \int_{0}^{2 \pi}\left[\varepsilon f_{0}(a, \psi)+\varepsilon^{2} f_{1}(a, \psi)\right] \cos \psi d \psi-\frac{\varepsilon}{2 \omega}\left[6 k^{1}-1\right] D_{1}^{2}(a),
\end{aligned}
$$

where

$$
D_{1}(a)=\frac{3 a^{2}}{8\left(2 k^{3}-k\right)} .
$$

In view of the fact that

$$
u_{1}(a, \psi)=\frac{a^{3}}{32\left(9 k^{4}-1\right)} \cos 3 \psi,
$$

according to (3.33), the function $f_{1}(a, \psi)$ can be written as follows:

$$
f_{1}(a, \psi)=3 a^{2} \cos ^{2} \psi u_{1}(a, \psi)-4 D_{1}(a) k^{3} u_{1 \psi \psi \psi \psi}(a, \psi)-2 D_{1}(a) k u_{1 \psi \psi}(a, \psi) .
$$


Taking into account that

$$
u_{1 \psi \psi}(a, \psi)=-\frac{9 a^{3}}{32\left(9 k^{4}-1\right)} \cos 3 \psi, \quad u_{1 \psi \psi \psi \psi}(a, \psi)=\frac{81 a^{3}}{32\left(9 k^{4}-1\right)} \cos 3 \psi
$$

we get

$$
f_{1}(a, \psi)=\frac{3 a^{5}}{32\left(9 k^{4}-1\right)} \cos ^{2} \psi \cos 3 \psi-\frac{3 a^{5} \cdot 18\left(18 k^{3}-k\right)}{8\left(2 k^{3}-k\right) 32\left(9 k^{4}-1\right)} \cos 3 \psi .
$$

Thus, it is easy to see that system (3.4) can be represented as follows:

$$
\begin{aligned}
& \frac{\partial a}{\partial t}+\omega^{\prime} \frac{\partial a}{\partial x}=0 \\
& \frac{\partial \varphi}{\partial t}+\omega^{\prime} \frac{\partial \varphi}{\partial x}=\frac{1}{2 \pi \omega a} \int_{0}^{2 \pi}\left[\varepsilon f_{0}(a, \psi)+\varepsilon^{2} f_{1}(a, \psi)\right] \cos \psi d \psi-\frac{\varepsilon^{2}}{2 \omega}\left(6 k^{2}-1\right) D_{1}^{2}(a) .
\end{aligned}
$$

Finally, we obtain a correction for the wave number $k$ in the second approximation in the following form:

$$
\frac{\partial \varphi}{\partial x}=\frac{3 \varepsilon a^{2}}{8\left(2 k^{3}-k\right)}+\varepsilon^{2} \frac{3 a^{4}}{128\left(2 k^{3}-k\right)}\left[\frac{1}{2\left(9 k^{4}-1\right)}+\frac{3\left(6 k^{2}-1\right)}{\left(2 k^{3}-k\right)^{2}}\right] .
$$

Let us describe a method that is successfully applied to various problems connected with the propagation of waves in liquids. The core of this method is the averaging of the Lagrangian and derivation of the corresponding Euler-Lagrange equations (already averaged). Sturrock [5], Whitham [4, 6-8], Bisshopp [9], and several other scientists developed this method under the assumption that, in the investigation of wave processes, the frequency, wave number, and amplitude can be re garded as slowly varying functions of space coordinates and time. In fact, these assumptions coincide with those made in the present paper but, in some cases, as is shown below, our method al lows one to obtain the corresponding results in the first approximation in a simpler way. However, it is worth noting that this method is not always applicable, because we cannot easily construct the corresponding Lagrangian for all equations. Furthermore, the right-hand sides of Eq. (3.1) considered in this paper give more possibilities for a detailed investigation of specific features of perturbed wave processes.

Below, we describe the application of this method to the model Bretherton equation (3.2), following the elegant presentation due to Nayfeh [10].

It easy to verify that this equation is the Euler-Lagrange equation that corresponds to the Lagrangian

$$
\mathcal{L}=\frac{1}{2} u_{t}^{2}-\frac{1}{2} u_{x x}^{2}+\frac{1}{2} u_{x}^{2}-\frac{1}{2} u^{2}+\frac{\varepsilon}{4} u^{4} .
$$

Indeed, according to the variation principle for Lagrangian (3.67), we obtain an EulerLagrange equation of the form

$$
\frac{\partial}{\partial t}\left(\frac{\partial L}{\partial u_{t}}\right)+\frac{\partial}{\partial x}\left(\frac{\partial L}{\partial u_{x}}\right)-\frac{\partial^{2}}{\partial x^{2}}\left(\frac{\partial L}{\partial u_{x x}}\right)-\frac{\partial L}{\partial u}=0 .
$$

By substituting expression (3.67) for the Lagrangian $\mathcal{L}$ in Eq. (3.68), we obtain Eq. (3.2). 
We seek a solution of Eq. (3.8), taking into account the form of its right-hand side, in the form of the expansion

$$
u(t, x)=a \cos \psi+\varepsilon \sum_{n=1}^{\infty} A_{n} \cos n \psi+\varepsilon^{2} \ldots,
$$

where, as before, $\psi=k x-\omega t+\varphi$, i.e., $\psi_{x}=k, \psi_{t}=-\omega$, and, consequently,

$$
k_{t}+\omega_{k}=0
$$

and the quantities $a, \omega, k$, and $A_{i}$ are slowly varying functions of $t$ and $x$.

Since, in the direct expansion, secular terms appear first among the terms of order $\varepsilon$, we assume that the quan tities $a_{x}, a_{t}, \omega_{x}, \omega_{t}, k_{x}$, and $k_{t}$ have the order $\varepsilon$.

Thus, we can write

$$
\begin{aligned}
& u_{t}=a \omega \sin \psi+a_{t} \cos \psi+\varepsilon \omega \sum_{n=2}^{\infty} n A_{n} \sin n \psi+\varepsilon^{2} \ldots, \\
& u_{x}=-a k \sin \psi+a_{x} \cos \psi-\varepsilon k \sum_{n=2}^{\infty} n A_{n} \sin n \psi+\varepsilon^{2} \ldots \\
& u_{x x}=-a k^{2} \cos \psi-2 a_{x} k \sin \psi-\varepsilon k^{2} \sum_{n=2}^{\infty} n^{2} A_{n} \cos n \psi+\varepsilon^{2} \ldots
\end{aligned}
$$

By substituting expressions (3.71) and (3.69) in Lagrangian (3.67), we obtain a Lagrangian that implicitly depends on $t$ and $x$ via the functions $\psi, a, \omega, k$, and $A_{1}$. The terms of this Lagrangian are $2 \pi$-periodic in $\psi$ and, as $\psi$ runs through the interval $[0,2 \pi]$, the changes in the other parameters are very small, whereas the Lagrangian varies much faster. Therefore, as is accepted in all modifications of the averaging method, the Lagrangian should be averaged with respect to $\psi$ over the interval $[0,2 \pi]$ under the assumption that the quantities $a, \omega, k$, and $A_{i}$ re main constant.

By averaging the right-hand sides of expressions (3.68) and (3.70), we obtain

$$
\begin{aligned}
\bar{u}^{4} & =\frac{3}{8} a^{4}+\varepsilon \ldots, \\
\bar{u}_{t}^{2} & =\frac{1}{2} a^{2} \omega^{2}+\varepsilon \ldots, \\
\bar{u}_{x}^{2} & =\frac{1}{2} a^{2} k^{2}+\varepsilon \ldots, \\
\bar{u}_{x x}^{2} & =\frac{1}{2} a^{2} k^{2}+\varepsilon \ldots .
\end{aligned}
$$

By substituting expressions (3.72) into the right-hand side of (3.67), we obtain the averaged Lagrangian

$$
\overline{\mathcal{L}}=\frac{1}{4}\left(\omega^{2}-k^{4}+k^{2}-1\right) a^{2}+\frac{3 \varepsilon}{32} a^{4}+\varepsilon^{2} \ldots,
$$

which explicitly depends on $a$ and implicitly on $\psi$ via $\omega$ and $k$ (according to expression (3.70) and the relations $\psi_{k}=k$ and $\psi_{t}=-\omega$ ). 
By using the averaged Lagrangian (3.73), we write the Euler-Lagrange equations (the averaged equations) that correspond to the variables $a$ and $\psi$. The Euler-Lagrange equation for $a$ has the form

$$
\frac{\partial \bar{L}}{\partial a}=0
$$

whence we immediately get the dispersion relation

$$
\omega^{2}=k^{4}-k^{2}+1+\frac{3 \varepsilon}{4} a^{2}+\varepsilon^{2} \ldots
$$

Thus, in the first approximation, the dispersion relation (3.75) obtained with the use of the averaged Lagrangian completely coincides with the dispersion relation (3.64) obtained by the KBM method.

By using expression (3.70) and the fact that $\psi_{k}=k$ and $\psi_{t}=-\omega$, we can write the Euler-Lagrange equation for the variable $\psi$ in the form

or

$$
\frac{\partial}{\partial t}\left[\frac{\partial \bar{L}}{\partial \psi_{t}}\right]+\frac{\partial}{\partial x}\left[\frac{\partial \bar{L}}{\partial \psi_{x}}\right]-\frac{\partial \bar{L}}{\partial \bar{\psi}}=0
$$

$$
-\frac{\partial}{\partial t}\left[\frac{\partial \bar{L}}{\partial \omega}\right]+\frac{\partial}{\partial x}\left[\frac{\partial \bar{L}}{\partial k}\right]=0
$$

By differentiating the right-hand side of (3.73) with respect to $\omega$ and $k$, we get

$$
\frac{\partial \bar{L}}{\partial \omega}=\frac{1}{2} \omega a^{2} \ldots, \quad \frac{\partial \bar{L}}{\partial k}=-\frac{1}{2}\left(2 k^{3}-k\right) a^{2}+\varepsilon^{2} \ldots
$$

We can now represent expression (3.77) in the form

$$
\frac{\partial}{\partial t}\left(\omega a^{2}\right)+\frac{\partial}{\partial x}\left[\left(2 k^{3}-k\right) a^{2}\right]=0
$$

or, taking into account the expression for the group velocity $\omega^{\prime}=d \omega / d k$ introduced above,

$$
\frac{\partial}{\partial t}\left[\omega a^{2}\right]+\frac{\partial}{\partial x}\left[\omega \omega^{\prime} a^{2}\right]=0
$$

or

$$
\omega\left[\frac{\partial a^{2}}{\partial t}+\frac{\partial}{\partial x}\left(\omega^{\prime} a^{2}\right)\right]+a^{2}\left[\omega_{t}+\omega^{\prime} \omega_{x}\right]=0
$$

Since $\omega=\omega(k)$, according to (3.75), we have $\omega_{t}=\omega^{\prime} k_{t}$ and, hence, in view of (3.70), $\omega_{t}+\omega^{\prime} \omega_{x}=0$ and Eq. (3.81) takes the form

$$
\frac{\partial a^{2}}{\partial t}+\frac{\partial}{\partial x}\left(\omega^{\prime} a^{2}\right)=0
$$

Furthermore, we can represent expression (3.70) in the form

$$
\frac{\partial k}{\partial t}+\omega^{\prime} \frac{\partial k}{\partial x}=0
$$

Thus, in the first approximation (to within quantities of order $\varepsilon$ ), for the solution of the Bretherton equation (3.2) $u=a \cos \psi(\psi=k x-\omega t)$, the dependence of the amplitude $a$, frequency $\omega$, and wave number $k$ on the space coordinates and time is determined by relations $(3.75),(3.82)$, and $(3.83)$. 
The first equation in system (3.26) obtained for the Bretherton equation (3.2) by the KBM method (under our assumptions concerning the physical meaning of the problem) coincides with Eq. (3.82). Furthermore, it is easy to show that the sum on the righthand side of expression (3.69) for the solution of Eq. (3.2) completely coincides with the expression for $u_{1}(a, \psi)$ obtained above [see formula (3.62)].

\section{REFERENCES}

1. F. P. Bretherton and C. J. R. Garrett, Wavetrain in inhomogeneous moving media, Proc. Roy. Soc. (London), A 302 (1967) 529-554.

2. N. N. Bogolyubov and Yu. A. Mitropol'skii, Asymptotic Methods in the Theory of Nonlinear Oscillations [in Russian], Nauka, Moscow, 1974.

3. Yu. A. Mitropol'skii, On the construction of an asymptotic solution of a perturbed Klein-Gordon equation, Ukr. Mat. Zh. 47 (3) (1995) 1209-1216.

4. D. Montgomery and D. A. Tidman, Secular and nonsecular behavior for the cold plasma equation, Phys. Fluids 7 (1964) 242-249.

5. P. A. Sturrock, A variational principle and an energy theorem for small amplitude disturbances of electron beams and of electron plasmas, Ann. Phys. 4 (1958) 306-324.

6. G. B. Whitham, A general approach to linear and nonlinear waves using a Lagrangian, J. Fluid Mech. 22 (1965) 273-283.

7. G. B. Whitham, Variational methods and application to water waves, Proc. Roy. Soc. (London), A 299 (1967) 6-25.

8. G. B. Whitham, Linear and Nonlinear Waves, Wiley, New York, 1974.

9. F. Bisshopp, A modified stationary principle for nonlinear waves, J. Different. Equat. 5 (1969) 592-605.

10. H. A. Nayfeh, Perturbation Methods, Wiley, New York, 1972.

Received June 30, 2007.

\section{XÂY DỰNG NGHIỆM TIỆM CẬN THEO NGHĨA \\ KRYLOV-BOGOLIUBOV-MITROPOLSKII CHO PHƯƠNG TRİNH SÓNG}

Trong bài báo đã nghiên cứu hai ví dụ áp dụng phương pháp KBM để xây dựng nghiệm gần đúng của phương trình Klein-Gordon-Bretherton hay gặp trong nhiều ứng dụng. 